\title{
In Vitro and In Vivo Efficacies of the Linear and the Cyclic Version of an All-D-Enantiomeric Peptide Developed for the Treatment of Alzheimer's Disease
}

\author{
Sarah Schemmert ${ }^{1}$, Luana Cristina Camargo ${ }^{1,2}$, Dominik Honold ${ }^{1}$, Ian Gering ${ }^{1} \mathbb{D}$, Janine Kutzsche ${ }^{1} \mathbb{D}$, \\ Antje Willuweit ${ }^{3}$ (D) and Dieter Willbold ${ }^{1,2, *(\mathbb{D})}$ \\ 1 Institute of Biological Information Processing, Structural Biochemistry (IBI-7), Forschungszentrum Jülich, \\ 52425 Jülich, Germany; s.schemmert@fz-juelich.de (S.S.); 1.camargo@fz-juelich.de (L.C.C.); \\ d.honold@fz-juelich.de (D.H.); i.gering@fz-juelich.de (I.G.); j.kutzsche@fz-juelich.de (J.K.) \\ 2 Institut für Physikalische Biologie, Heinrich-Heine-Universität Düsseldorf, 40225 Düsseldorf, Germany \\ 3 Institute of Neuroscience and Medicine, Medical Imaging Physics (INM-4), Forschungszentrum Jülich, \\ 52425 Jülich, Germany; a.willuweit@fz-juelich.de \\ * Correspondence: d.willbold@fz-juelich.de; Tel.: +49-246-161-2100
}

\section{check for}

updates

Citation: Schemmert, S.; Camargo, L.C.; Honold, D.; Gering, I.; Kutzsche,

J.; Willuweit, A.; Willbold, D. In Vitro and In Vivo Efficacies of the Linear and the Cyclic Version of an All-D-Enantiomeric Peptide Developed for the Treatment of Alzheimer's Disease. Int. J. Mol. Sci. 2021, 22, 6553. https://doi.org/ $10.3390 /$ ijms 22126553

Academic Editor: Sónia C. Correia

Received: 21 May 2021

Accepted: 16 June 2021

Published: 18 June 2021

Publisher's Note: MDPI stays neutral with regard to jurisdictional claims in published maps and institutional affiliations.

Copyright: (C) 2021 by the authors Licensee MDPI, Basel, Switzerland. This article is an open access article distributed under the terms and conditions of the Creative Commons Attribution (CC BY) license (https:// creativecommons.org/licenses/by/ $4.0 /)$
Abstract: Multiple sources of evidence suggest that soluble amyloid $\beta$ (A $\beta$ )-oligomers are responsible for the development and progression of Alzheimer's disease (AD). In order to specifically eliminate these toxic $\mathrm{A} \beta$-oligomers, our group has developed a variety of all-D-peptides over the past years. One of them, RD2, has been intensively studied and showed such convincing in vitro and in vivo properties that it is currently in clinical trials. In order to further optimize the compounds and to elucidate the characteristics of therapeutic D-peptides, several rational drug design approaches have been performed. Two of these D-peptides are the linear tandem (head-to-tail) D-peptide RD2D3 and its cyclized form cRD2D3. Tandemization and cyclization should result in an increased in vitro potency and increase pharmacokinetic properties, especially crossing the blood-brain-barrier. In comparison, cRD2D3 showed a superior pharmacokinetic profile to RD2D3. This fact suggests that higher efficacy can be achieved in vivo at equally administered concentrations. To prove this hypothesis, we first established the in vitro profile of both D-peptides here. Subsequently, we performed an intraperitoneal treatment study. This study failed to provide evidence that cRD2D3 is superior to RD2D3 in vivo as in some tests cRD2D3 failed to show equal or higher efficacy.

Keywords: Alzheimer's disease; D-peptides; treatment; behavior; Tg-SwDI mice; cognition; disassembly; $A \beta$; oligomers

\section{Introduction}

Our society is aging and, with it, the number of diseases of old age, especially dementias, are increasing [1]. Alzheimer's disease (AD) is a devastating neurodegenerative disorder and the most common form of dementia worldwide. Its clinical symptoms are considered to be disturbances of memory, language, spatial and temporal orientation and cognitive decline. The pathological hallmarks of the disease are characterized by neurodegeneration, intracellular depositions of neurofibrillary tangles and extracellular accumulations of amyloid- $\beta(A \beta)$ plaques. $A \beta$ is the product of the proteolytic processing of the amyloid precursor protein (APP), which is cleaved by $\beta$ - and $\gamma$-secretases, resulting in $A \beta$-monomers. Due to unknown reasons, these monomers aggregate into $A \beta$-oligomers and insoluble $A \beta$-fibrils, which compact further into senile $A \beta$-plaques [2,3]. For a long time, it was assumed that $A \beta$-plaques were responsible for the disease and the cognitive decline of affected patients. Currently, however, the aforementioned soluble $A \beta$-oligomers are regarded as more than merely an intermediate on their way from monomers to plaques. Instead, they are postulated to be the most toxic species, responsible for synapse deterioration, neuronal death, disrupted $\mathrm{Ca}^{2+}$-homeostasis and dysfunctional plasticity-all in all 
leading to the clinical symptoms of $\mathrm{AD}$ [4]. In addition, there is increasing evidence that $A \beta$-oligomers are able to replicate in a prion-like fashion [5]. In our group, we are focused on the development of compounds for a disease modifying or even curative treatment of AD. For this purpose, we designed all-D-enantiomeric peptides to directly destabilize, disassemble and ultimately eliminate toxic $\mathrm{A} \beta$-oligomers via direct disruption, rather than relying on the immune system for their degradation. By use of mirror image phage display against monomeric $A \beta$, the lead compound $D 3$, a D-enantiomeric peptide consisting of 12 amino acid residues, arose [6]. D-peptides have several advantages as therapeutics compared to their L-enantiomeric equivalents. As shown by our group and others, the proteolytic stability of D-peptides is superior to L-peptides with the same sequence of amino acid residues, because proteases are most often stereoselective for L-amino acid residues [7]. This results also in a reduced immunogenicity and increased bioavailability of D-peptides [8]. In order to optimize the lead structure, with respect to increased stability, affinity to $A \beta(1-42)$, blood-brain-barrier (BBB) penetration, and in vitro potency and in vivo efficacy, rational drug design approaches were conducted. The most promising compound so far, called RD2, is currently under development for the treatment of patients with AD [9]. The suggested mode of action was demonstrated by successful target engagement in vitro and in vivo [10,11]. Furthermore, the in vivo efficacy of RD2 was proven in different AD mouse models, even in old-aged mice with fully developed AD-associated pathology [10-13]. Besides RD2, more D-peptides were developed out of a rational drug design. In order to combine the favorable properties of D3 and RD2, a heteromeric linear head-to-tail version of both D-peptides, called RD2D3, was designed. Compared to RD2 and $D 3$, we have shown increased binding affinity to $A \beta(1-42)[14]$ and a higher potency to eliminate toxic A $\beta$-oligomers for RD2D3 [12]. In vivo, it was demonstrated that RD2D3 has a favorable pharmacokinetic profile (after intraperitoneal (i.p.) administration), high proteolytic stability and therapeutic efficacy by improving the cognitive abilities of transgenic APP Swedish/Dutch/Iowa (Tg-SwDI) AD mice [14,15]. Besides the development of linear tandem-D-peptides, a further approach for a rational drug design was the cyclization of either homo- or tandem-D-peptides [16,17]. For the cyclized tandem-D-peptide cRD2D3, a remarkably enhanced pharmacokinetic profile was found [17]. Comparing the pharmacokinetic profiles of the linear and cyclic version of the D-peptide RD2D3 (RD2D3 vs. cRD2D3) after intravenous (i.v.). and intraperitoneal (i.p.) administration in C57Bl/ 6 wild type (WT) mice resulted in a tremendously increased terminal half-life ( $2.3 \mathrm{vs.} 58 \mathrm{~h})$, increased BBB penetration values and brain peptide concentrations of cRD2D3. Furthermore, for cRD2D3 a high oral bioavailability was demonstrated [17]. Both, a long half-life and high oral bioavailability are extremely advantageous for active ingredients used in the therapy of $\mathrm{AD}$ and qualify for daily drug administrations. Since it can be assumed that AD therapy will take place over a longer period of time (possibly several years to decades), a therapy regime with once-daily oral drug intake is most convenient for the patient (treatment adherence).

Here, we wanted to investigate whether the more favorable pharmacokinetic profile of the cyclic D-peptide cRD2D3 compared to its linear version RD2D3 results in an increased in vivo efficacy. Before investigating the in vivo efficacy of RD2D3 and cRD2D3 in the Tg-SwDI AD mouse model, we performed an in-depth in vitro characterization of the compounds on the specific $A \beta$-mutation, which was introduced to the Tg-SwDI AD mouse model in comparison to wild type $A \beta(1-42)$. This $A \beta$ mutation is the so called "Dutch" and "Iowa" mutation $(D / I A \beta)$ and it is described by an exchange of the amino acid glutamate (E) for glutamine $(\mathrm{Q})$ at position 22 and of aspartate $(\mathrm{D})$ for asparagine $(\mathrm{N})$ at position 23 of the $\mathrm{A} \beta(1-42)$ sequence.

\section{Results}

2.1. The Cyclic D-Peptide cRD2D3 Revealed Increased In Vitro Potency Compared to the Linear D-Peptide RD2D3 Without Differences in the Proteolytic Stability

In order to investigate the in vitro potency of the cyclic D-peptide cRD2D3 and its linear form RD2D3, we performed several experiments concerning the binding of the D-peptides to $A \beta(1-42)$ and $D / I A \beta$. By use of surface plasmon resonance (SPR) mea- 
surements, we determined the dissociation constant $\left(\mathrm{K}_{\mathrm{D}}\right)$ of RD2D3 and cRD2D3 to both $A \beta$-species, by using $A \beta$ as ligand and the D-peptide as analyte or vice versa. As illustrated in Figure 1, both D-peptides bound with $\mu \mathrm{M}$ concentrations to $\mathrm{A} \beta(1-42)$ and to $\mathrm{D} / \mathrm{I} A \beta$. By use of $\mathrm{A} \beta(1-42)$ or $\mathrm{D} / \mathrm{I} \mathrm{A} \beta$ as an analyte, both D-peptides bound with higher affinity to D/I A $\beta\left(K_{D} \mathrm{~A} \beta(1-42)\right.$ : RD2D3 $7.01 \pm 0.31 \mu \mathrm{M}$, cRD2D3 $10.41 \pm 0.61 \mu \mathrm{M}$; D/I $\mathrm{A} \beta$ : RD2D3 $3.24 \pm 0.31 \mu \mathrm{M}, \mathrm{cRD} 2 \mathrm{D} 32.05 \pm 0.33$, Figure 1). By use of the D-peptides as a ligand, cRD2D3 bound with an almost identical affinity to both $A \beta$ species $\left(K_{D} A \beta(1-42)\right.$ : $5.63 \pm 2.5 \mu \mathrm{M}, \mathrm{K}_{\mathrm{D}} \mathrm{D} / \mathrm{I} \mathrm{A} \beta 5.99 \pm 0.77 \mu \mathrm{M}$, Figure 1). For RD2D3, the picture is different. The D-peptide bound with half the affinity to $A \beta(1-42)$ than to $D / I A \beta\left(K_{D} A \beta(1-42)\right.$ : $18.4 \pm 6.5 \mu \mathrm{M}, \mathrm{K}_{\mathrm{D}} \mathrm{D} / \mathrm{I} \mathrm{A} \beta 7.09 \pm 1.4 \mu \mathrm{M}$, Figure 1$)$.
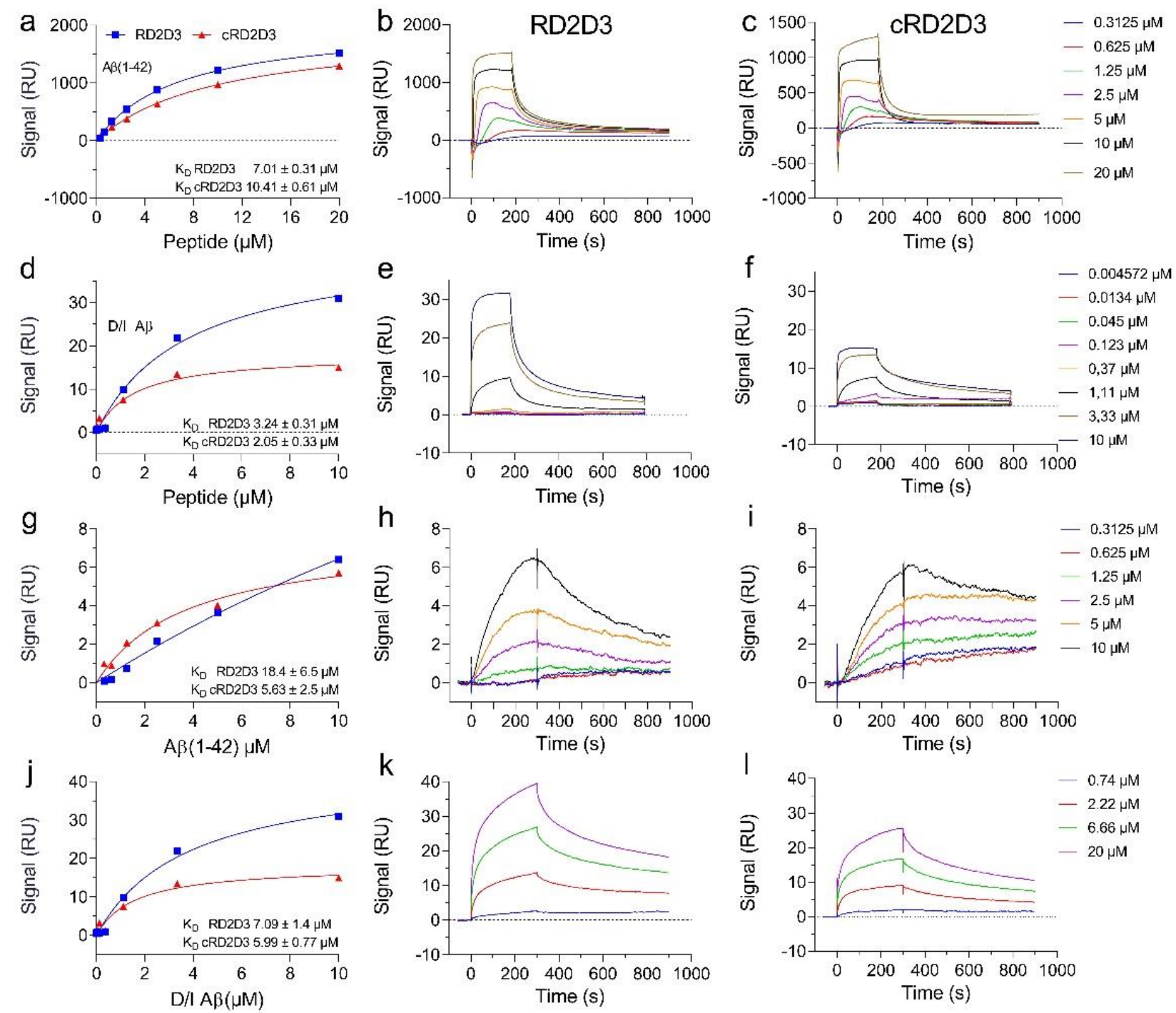

Figure 1. Affinity determination of RD2D3 and cRD2D3 to A $\beta(1-42)$ and D/I A $\beta$ by SPR. By use of the D-peptides as analyte, either $A \beta(1-42)$ or $D / I A \beta$ was immobilized on a CM-5 sensor chip and the binding of different RD2D3 (blue) and cRD2D3 (red) concentrations were analyzed in a multi cycle experiment (a-f). Real-time surface plasmon resonance (SPR) sensorgrams of different RD2D3 and cRD2D3 concentrations (left). Equilibrium dissociation constants ( $\mathrm{K}_{\mathrm{D}}$ ) were determined by using a Langmuir 1:1 binding model (RD2D3 middle, cRD2D3 right). RD2D3 or cRD2D3 were immobilized on a series S CM-5 sensor chip, and the binding of $A \beta(1-42)$ or $D / I A \beta$ monomers as an analyte at various concentrations was observed (g-1). For evaluation, the steady-state binding signals were plotted over the concentrations and fitted using a Langmuir 1:1 binding model (a,d,g,j). 
The ability to inhibit the fibril formation of $A \beta$ is of great importance for substances developed for a treatment of AD. In order to test for interference with fibril formation, we performed a thioflavin T test (ThT). The fluorescence signal of ThT, a benzothiazole dye, increases upon binding to amyloid fibrils. In order to investigate the functional effects of RD2D3 and cRD2D3 on $A \beta(1-42)$ and on $D / I A \beta$, the D-peptides were incubated with either $A \beta(1-42)$ or $D / I A \beta$ (equimolar concentrations) and $5 \mu \mathrm{M}$ ThT. ThT fluorescence was monitored every $6 \mathrm{~min}$ for $24 \mathrm{~h}$. As demonstrated in Figure 2a,b, both D-peptides were able to inhibit the $A \beta(1-42)$ and $D / I A \beta$ fibril formation. Interestingly, this effect was more pronounced on $A \beta(1-42)$ than on $D / I A \beta$ (Figure 2a,b). Besides the potency of RD2D3 and cRD2D3 to inhibit the $A \beta$ fibril formation, we also investigated the potency of both compounds to eliminate toxic $A \beta$-oligomers. For this, the so called QIAD (quantitative determination of interference with $A \beta$ aggregate size distribution) assay [18] was conducted with both D-peptides and $A \beta(1-42)$ and $D / I A \beta$. The outcome of the QIAD assay revealed that both RD2D3 and cRD2D3 were capable to significantly eliminate toxic $A \beta(1-42)-$ oligomers (RD2D3 89\%, cRD2D3 80\%, Figure 2c, one-way ANOVA, with Bonferroni post hoc analysis, $\mathrm{A} \beta(1-42)$ vs. RD2D3 fraction $4 p=0.001$, fraction $5, p=0.011$, fraction $6 p=0.01$, cRD2D3 fraction $4 p=0.005$, fraction $5 p=0.12$, fraction $6 p=$ n.s. (0.057)). Furthermore, both D-peptides eliminated toxic D/I A $\beta$-oligomers with a similar potency (RD2D3 94\%, cRD2D3 100\%, Figure 2d, not significant for cRD2D3 despite the total elimination of toxic oligomers, one-way ANOVA with Bonferroni post hoc analysis, D/I A $\beta$ vs. RD2D3 fraction $4 p=$ n.s.(0.062), fraction 5, $p=0.011$, fraction $6 p=$ n.s, cRD2D3 fraction $4 p=$ n.s. (0.094), fraction $5 p=$ (n.s.) 0.118 and fraction $6 p=$ n.s.).

In order to verify and extend the proteolytic stability/in vitro ADME (absorption, distribution, metabolism and excretion) of RD2D3 and cRD2D3, previously described for tritium labeled D-peptides $\left({ }^{3} \mathrm{H}\right)[15,17]$, we performed different tests to investigate the proteolytic stability in different (simulated) body fluids. In both, simulated gastric (SGF) and intestinal fluid (SIF), RD2D3 and cRD2D3 were remarkably stable (RD2D3: SIF $4 \mathrm{~h}$ $100 \%$, 8 h $93.5 \%$, SGF 4 h $100 \%$, 8 h $100 \%$, cRD2D3: SIF 4 h $96 \%$, 8 h $87.2 \%$, SGF 4 h $99.5 \%$

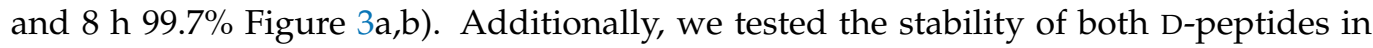
human plasma and human liver microsomes. In plasma, both D-peptides were stable up to approximately $90 \%$ after $48 \mathrm{~h}$ (RD2D3: 8 h 84.7\%, $24 \mathrm{~h} \mathrm{91.9 \%} \mathrm{and} 48 \mathrm{~h} \mathrm{93.7 \%} \mathrm{and} \mathrm{cRD2D3:}$

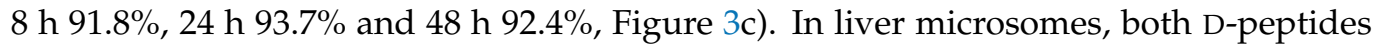
were slightly metabolized to approximately $25 \%$ (RD2D $3: 8 \mathrm{~h} 81.9 \%$ and $24 \mathrm{~h} 78.2 \%$ and cRD2D3: 8 h $89.2 \%$ and 24 h $73.6 \%$, Figure 3d).

\subsection{Tg-SwDI Mice Develop Cognitive Deficits at 12 Months of Age Compared to WT Mice}

In all conducted experiments (nesting behavior, marble burying, open field test and Morris water maze (MWM)) Tg-SwDI mice showed an altered behavior compared to WT mice, but there was no significant difference in the body weight of WT compared to Tg-SwDI mice (Figure 4a). Analyses of some basic behavioral characteristics of Tg-SwDI resulted in phenotypic alterations. Compared to WT mice, Tg-SwDI mice formed a less mature nest (unpaired two-tailed $t$ test, $p<0.001$, Figure $4 \mathrm{~b}$ ). Furthermore, they displayed impaired digging behavior in direct comparison to WT mice of the same age, indicating a less inquisitive behavior (unpaired two-tailed $t$ test, $p<0.001$, Figure $4 \mathrm{c}$ ). 

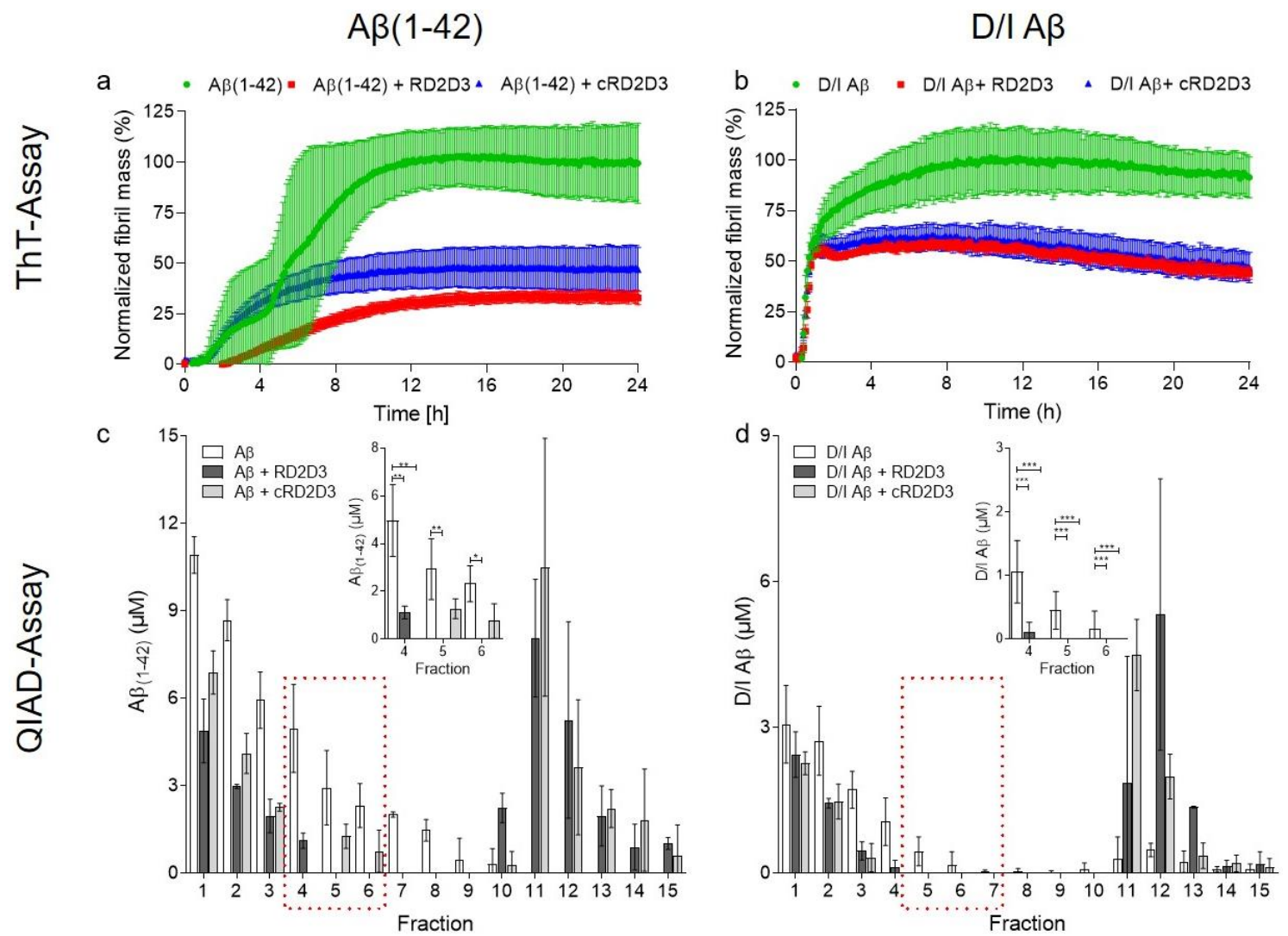

Figure 2. RD2D3 and $c R D 2 D 3$ inhibited the $A \beta(1-42)$ and $D / I A \beta$ fibril formation and eliminated significantly toxic oligomers. ThT-Assay (upper panel). Both D-peptides, RD2D3 (red) and cRD2D3 (blue), were analyzed according to their ability to inhibit either the $A \beta(1-42)$ (a,left) or the $D / I A \beta$ (b,right) fibril formation by use of equimolar concentrations $(10 \mu \mathrm{M})$. All analyzed D-peptides were able to inhibit either the A $\beta(1-42)$ (a) or the D/I A $\beta$ (both green) (b) fibril formation. Fibril mass was normalized to the $\mathrm{A} \beta$ control. Data are presented as mean $\pm \operatorname{SD}(N=3$ out of three independent experiments). QIAD-Assay (lower panel). A $\beta(1-42)$ (c,left) and D/I A $\beta$ (d,right) size distribution without (white) or with D-peptide were analyzed by density gradient centrifugation with subsequent measurements of the $A \beta$ concentrations. $A \beta$-oligomers are located in fractions 4-6. Comparison of $20 \mu \mathrm{M}$ RD2D3 (dark grey) and $20 \mu \mathrm{M}$ cRD2D3 (light grey) (c) revealed similar $\mathrm{A} \beta(1-42)(80 \mu \mathrm{M})$ oligomer elimination efficacy of both D-peptides. Comparison of $10 \mu \mathrm{M}$ RD2D3 (dark grey) and $10 \mu \mathrm{M}$ cRD2D3 (light grey) (d) revealed higher potency of RD2D3 to eliminate toxic D/I A $\beta(40 \mu \mathrm{M})$-oligomers than cRD2D3 does. Data are presented as mean $\pm \mathrm{SD}(N=2-5)^{* * *} p<0.001{ }^{* *} p<0.01$ and ${ }^{*} p<0.05$.
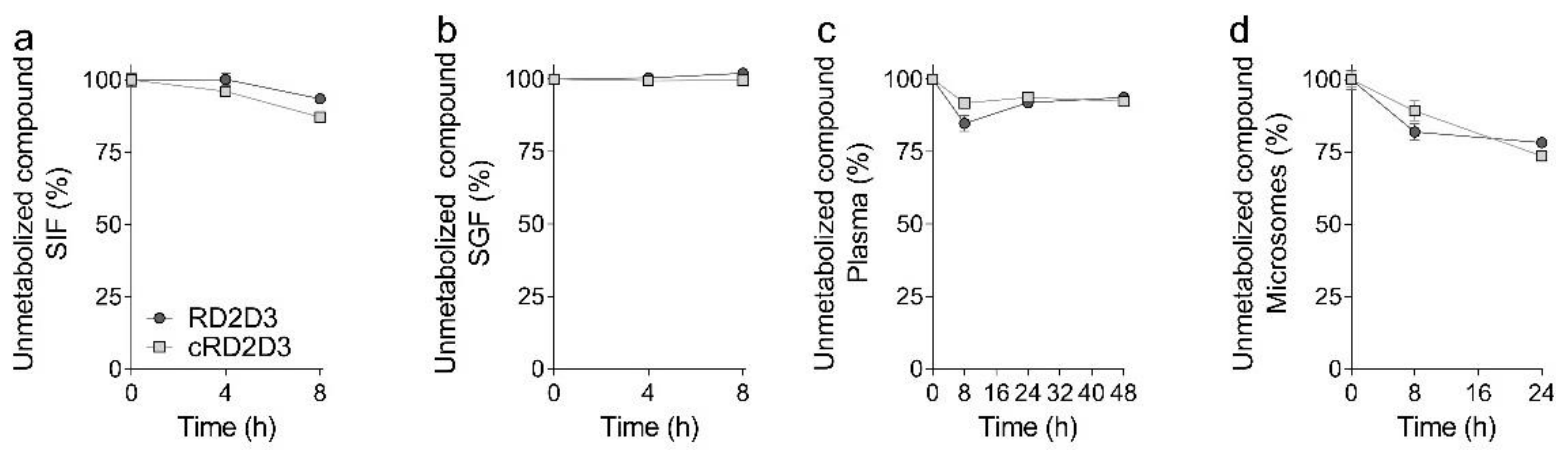

Figure 3. Proteolytic stability of RD2D3 and cRD2D3 in different human (simulated) body fluids. Both D-peptides, RD2D3 and cRD2D3, are remarkably stable in simulated intestinal and gastric fluid (SIF and SGF) (a,b), and in human plasma (c) and human liver microsomes (d). Shown are data form three independent experiments (mean $\pm \mathrm{SD}$ ). 

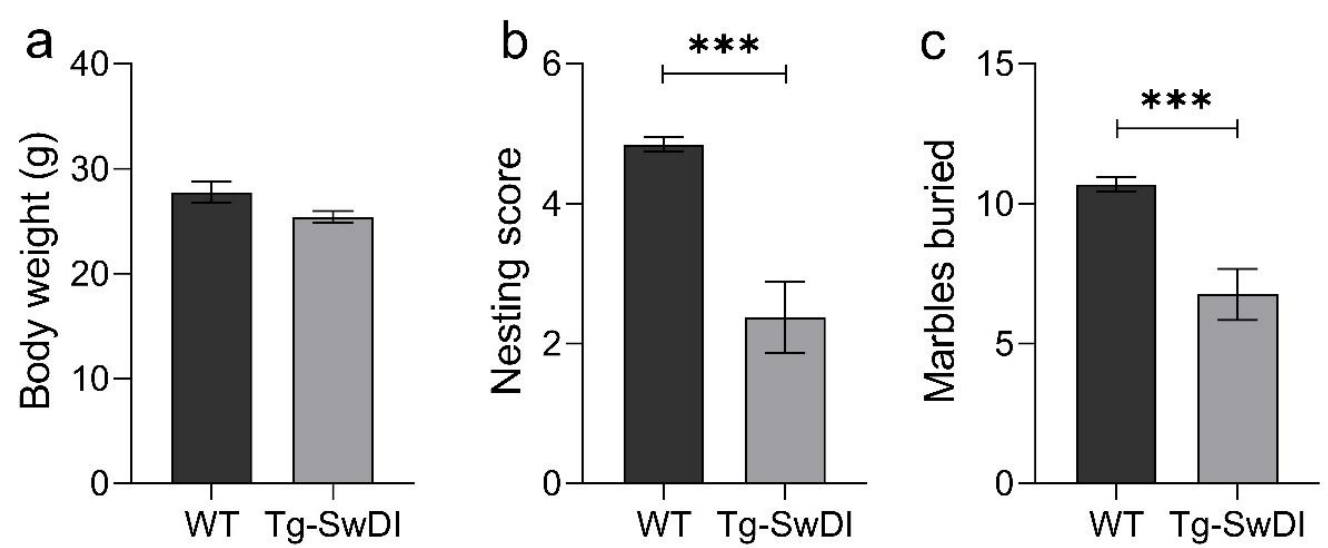

Figure 4. Basic phenotypic analysis of Tg-SwDI mice. No difference in body weight between Wild type (WT) and Tg-SwDI mice (a), but significant differences in nesting behavior (b) and marble burying (c) were found. Tg-SwDI mice formed a less mature nest and buried less marbles. Data are represented as mean $\pm \mathrm{SEM}$, all unpaired two-tailed $t$ test, ${ }^{* * *} p<0.001$ (WT $N=13$ and Tg-SwDI $N=12)$.

While performing the open field test, a significant difference was found between the time WT and Tg-SwDI spent in the center and border zone (two-way ANOVA genotypes $p<0.0001$, Fisher LSD post hoc analysis WT vs. Tg-SwDI center and border $p<0.0001$, Figure 5a). By analyzing the travelled duration, Tg-SwDI covered an almost two-times longer distance than WT mice (two-tailed t-test, $p<0.0001$, Figure 5b). Furthermore, TgSwDI mice were moving much faster than WT mice (two-tailed t-test, $p<0.0001$, Figure $5 c$ ). Moreover, by analysis of single time slots (each slot $5 \mathrm{~min}$ ) WT mice showed a clear habituation effect to the arena (Figure 5d). At the beginning of the test period, the center had an aversive effect to the mice, since they tended to avoid open areas. After a while, the WT mice explored the center of the arena more and more. In contrast, Tg-SwDI mice do not show this habituation effect (two-way RM ANOVA, genotype $p=0.002$, Fisher LSD post hoc analysis, WT vs. Tg-SwDI slot one $p=0.047$, slot two n.s. $(p=0.052)$, slot three $p=0.034$, slot four $p<0.014$ and slot five $p<0.001)$.

In order to analyze spatial memory, we conducted an MWM. On the first day of training, WT and Tg-SwDI mice performed almost equally (Figure 5e,f). Starting at day two, WT mice found the hidden platform significant faster than Tg-SwDI mice (two-way RM ANOVA, genotype $p=0.001$, Fisher LSD post hoc analysis, WT vs. Tg-SwDI day one n.s. $(p=0.91)$, day two $p=0.018$, day three $p=0.091$, day four $p<0.001$ and day five $p=0.004$, Figure 5e). A slight learning effect could be seen for both WT and Tg-SwDI mice. However, this effect was much more pronounced in the WT mice. During the probe trial, WT mice spent more time in the platform zone than Tg-SwDI mice, indicating an improved memory retrieval (two-tailed t-test, $p=0.073$, Figure $5 \mathrm{f}$ ). Concluding, Tg-SwDI mice developed distinct cognitive deficits as detected by the MWM with 12 months of age.

\subsection{RD2D3 Showed Superior Efficacy to Improve Phenotypic Deficits over cRD2D3}

All mice, regardless of treatment, showed no changes in their general appearance. Compared to D-peptide treated mice, placebo treated mice displayed a decrease in body weight (before vs. after treatment: placebo $35.1 \pm 0.5 \mathrm{~g}$ vs. $32.3 \pm 0.4 \mathrm{~g}$, RD2D3 $33.2 \pm 2.6 \mathrm{~g}$ vs. $34.3 \pm 0.6 \mathrm{~g}$ and cRD2D3 $35.1 \pm 1.4 \mathrm{~g}$ vs. $33.2 \pm 1.0 \mathrm{~g}$ ), but not to a significant extent (Figure 6a). There was no difference in the behavior of RD2D3 or cRD2D3 treated mice compared to placebo treated mice, neither in the nesting behavior, nor in the marble burying test. (Figure 6b,c). 

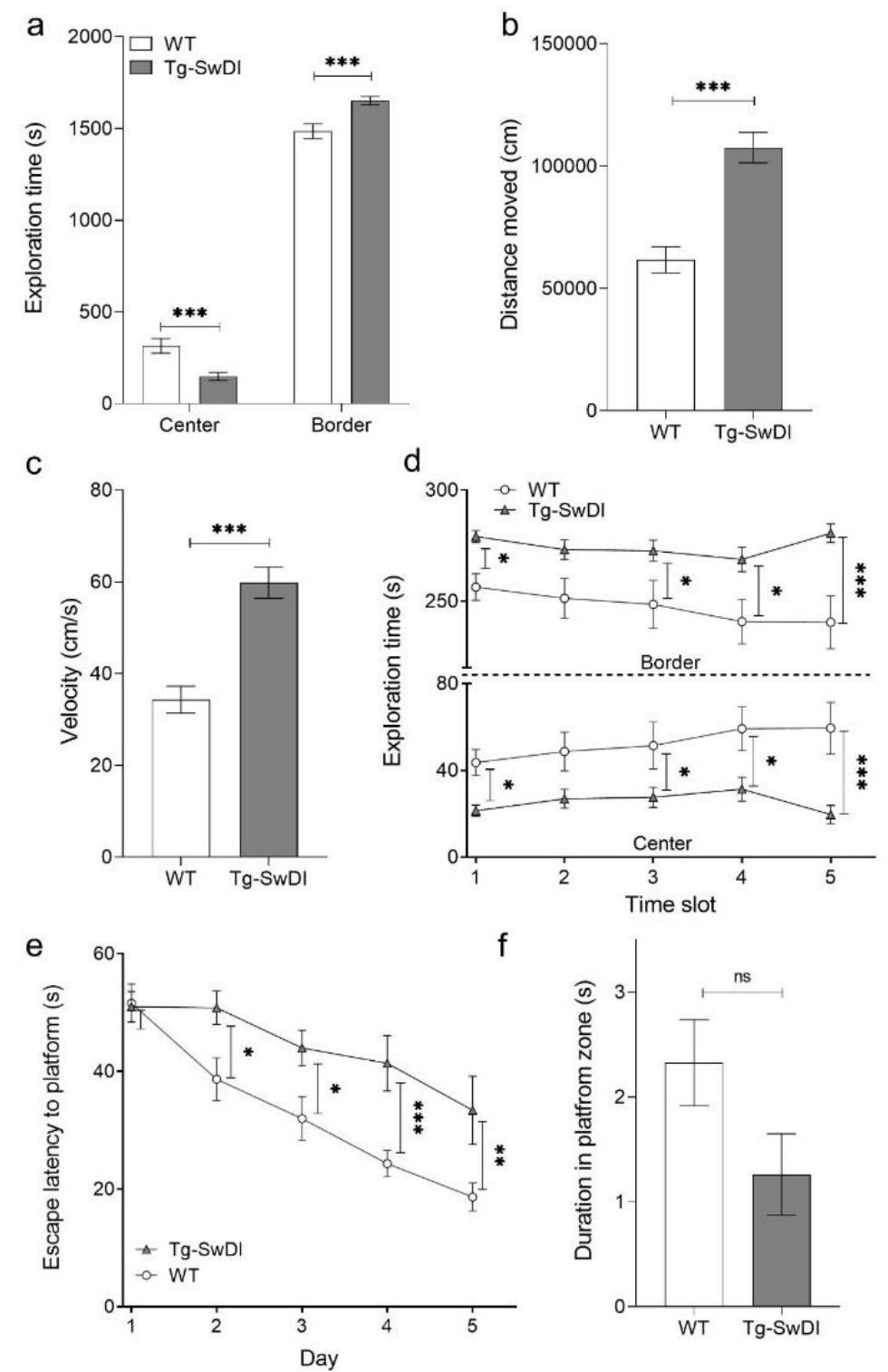

Figure 5. Tg-SwDI mice developed phenotypic deficits in the open field test and showed cognitive spatial impairment in the Morris water maze. In order to analyze some general aspects of the behavior of Tg-SwDI compared to wild type (WT) mice, an open field test was conducted, where mice were allowed to explore a square shaped arena freely for $30 \mathrm{~min}$. The analysis of the test revealed that Tg-SwDI mice explored less the center of the arena (a) (two-way ANOVA genotypes $p<0.0001$, Fisher LSD post hoc analysis WT vs. Tg-SwDI center and border $p<0.0001)$. Furthermore, they showed a reduced covered distance (b) and a reduced velocity (c) (both two-tailed $t$-test $p=0.0001$ ). By analyzing the habituation effect of WT and Tg-SwDI mice to the arena, it was obvious that transgenic mice explored the center of the arena less than WT mice, indicating a reduced habituation effect to the arena (d) (two-way RM ANOVA, genotype $p=0.002$, Fisher LSD post hoc analysis, WT vs. Tg-SwDI slot one $p=0.047$, slot two n.s. $(p=0.052)$, slot three $p=0.034$, slot four $p<0.014$ and slot five $p<0.001$ ). In the Morris water maze, a significant difference was detectable in the performance during the training of WT and Tg-SwDI mice starting at day 2, indicating spatial memory deficits (e) (two-way RM ANOVA, genotype $p=0.001$, Fisher LSD post hoc analysis, WT vs. Tg-SwDI day one n.s. $(p=0.91)$, day two $p=0.018$, day three $p=0.091$, day four $p<0.001$ and day five $p=0.004)$ ). During the probe trial, WT mice spent more time in the platform zone than Tg-SwDI mice, indicating impairments with memory retrieval (f). Data are shown as mean $\pm \mathrm{SEM}$, ${ }^{*} p<0.05,{ }^{* *} p<0.01$ and *** $p<0.001$ (WT $N=13$ and Tg-SwDI $N=12$ ). 

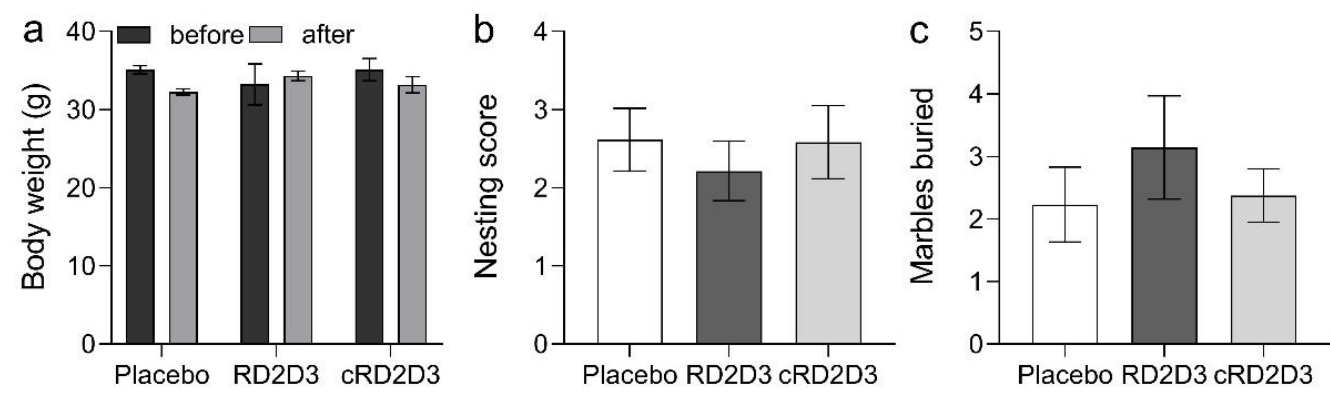

Figure 6. General behavior of RD2D3 and cRD2D3 treated tg-SwDI mice compared to placebo. There were no significant differences in all analyzed groups, neither in the body weight (a) nor in the nesting behavior $(\mathbf{b})$ or marble burying $(\mathbf{c})$. Data are represented as mean $\pm \mathrm{SEM}$ (placebo $N=13$, RD2D3 $N=14$ and cRD2D3 $N=12$ ).

During the total open field test duration, no difference between treated or non-treated Tg-SwDI was detectable (Figure 7). Neither in the time the treatment groups spent in the center or border zone (Figure 7a) nor in the covered distance or in the velocity (Figure $7 \mathrm{~b}, \mathrm{c}$ ). Taken a closer look, it was also possible to analyze the habituation effect of all mice to the arena. RD2D3 treated mice showed a slight but significant habituation effect to the arena compared to placebo treated mice (two-way RM ANOVA, $p=0.056$, Fisher LSD post hoc analysis, placebo vs. RD2D3 slot 1 n.s., slot $2 p=0.045$, slot 3 n.s., slot 4 n.s. and slot 5 $p=0.44)$, this effect was completely absent in cRD2D3 treated mice. During each analyzed time slot, they spent almost the same time in the center or border, respectively (Figure 7d).

In this study, a MWM was performed to investigate, whether a treatment with RD2D3 or cRD2D3 improves the spatial memory or cognitive abilities of Tg-SwDI mice compared to placebo treated mice. As can be seen from Figure 7e, both compounds were able to improve the performance of Tg-SwDI mice in the MWM during the training phase. For RD2D3, this effect was statistically significant compared to placebo treated mice (two-way RM ANOVA treatment $p=0.11$, Fisher post-hoc analysis placebo vs. RD2D3 day 1 n.s., day 2 n.n, day $3 p=0.004$, day 4 n.s. and day $5 p=0.029$ ). In order to test memory retrieval, a probe trial was conducted. Although not to a significant extent, there was a preference of RD2D3 treated mice spending more time in the platform zone (Figure 7f).

In order to investigate, whether treatment with RD2D3 or cRD2D3 change the pathological characteristics of Tg-SwDI mice, several histological analyses were conducted. As demonstrated in Table 1 and Figure 8, neither RD2D3 nor cRD2D3 were able to reduce the amount of $A \beta$ deposits as shown by $6 \mathrm{E} 10$ staining, nor reduce the number of activated astrocytes or microglia, as shown by GFAP or Iba-1 staining, respectively.

Table 1. Immunohistochemical investigations of RD2D3 and cRD2D3 treatment on A $\beta$ deposits and neuroinflammation. Treatment with neither RD2D3, nor cRD2D3 did reveal any increase or decrease in A $\beta$ deposits (6E10), activated astrocytes (GFAP) or microglia (Iba-1). IR: immunoreactivity. Data are represented as mean \pm SEM. IR: immunoreactivity (placebo $N=13, \operatorname{RD} 2 \mathrm{D} 3 \mathrm{~N}=14$ and $\mathrm{cRD} 2 \mathrm{D} 3 \mathrm{~N}=12$ ).

\begin{tabular}{ccccccc}
\hline & \multicolumn{2}{c}{ 6E10 IR (\%) } & \multicolumn{2}{c}{ GFAP IR (\%) } & \multicolumn{2}{c}{ Iba-1 IR (\%) } \\
\cline { 2 - 7 } & Cortex & Hippocampus & Cortex & Hippocampus & Cortex & Hippocampus \\
\hline Placebo & $1.8 \pm 0.7$ & $2.5 \pm 0.7$ & $43.8 \pm 2.0$ & $39.8 \pm 1.0$ & $8.7 \pm 1.7$ & $11.5 \pm 1.1$ \\
RD2D3 & $1.0 \pm 0.2$ & $2.5 \pm 0.5$ & $40.6 \pm 2.2$ & $38.0 \pm 1.2$ & $8.1 \pm 1.6$ & $8.6 \pm 1.3$ \\
cRD2D3 & $1.1 \pm 0.2$ & $4.2 \pm 0.7$ & $43.1 \pm 1.8$ & $39.5 \pm 1.0$ & $8.1 \pm 1.6$ & $10.2 \pm 1.8$ \\
\hline
\end{tabular}



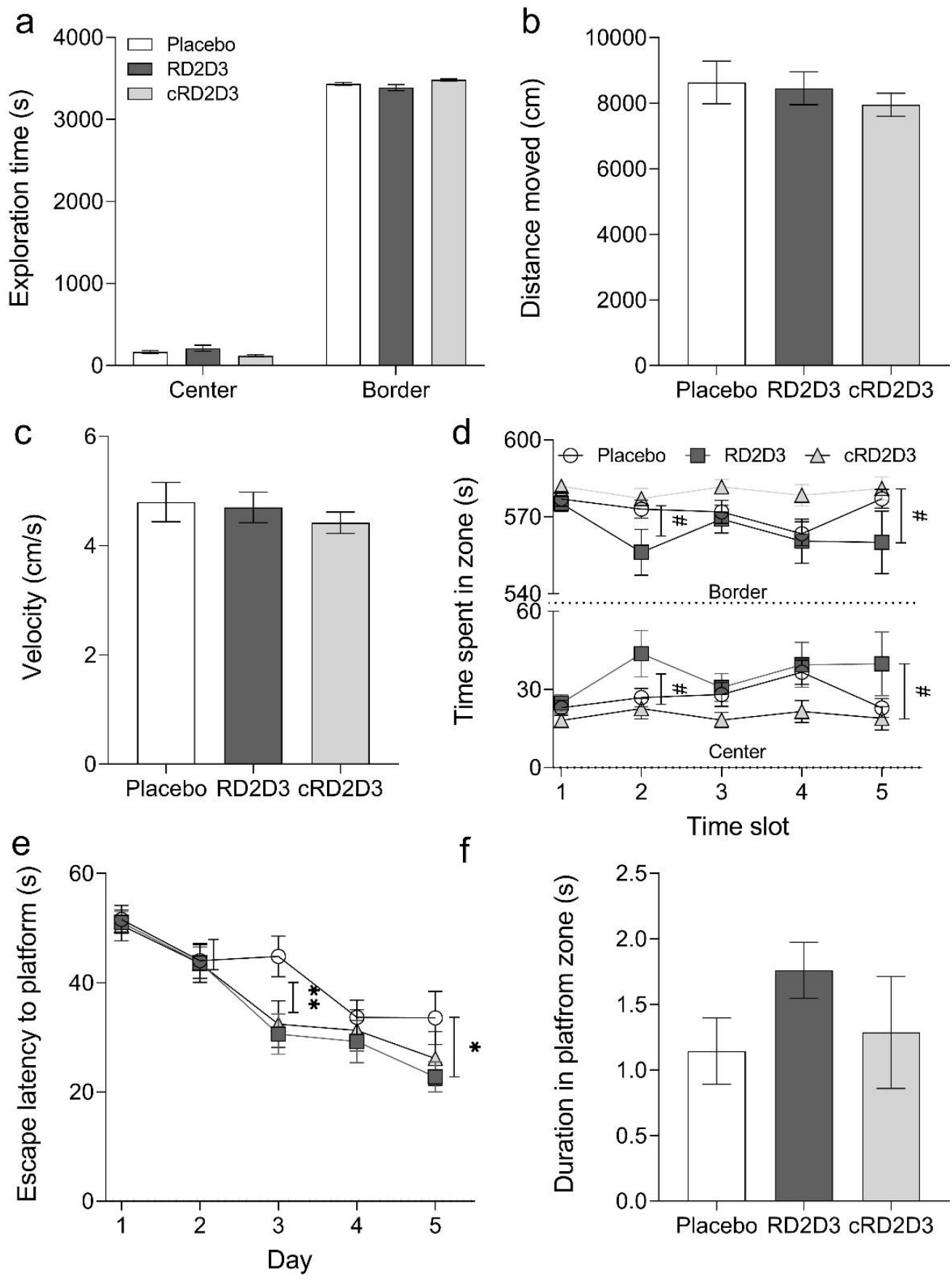

Figure 7. Treatment with RD2D3 significantly improved the phenotype of Tg-SwDI mice compared to placebo and cRD2D3 treated mice. An open field test was conducted to analyze and compare the exploratory and anxiety related behavior of RD2D3 and cRD2D3 treated Tg-SwDI mice compared to placebo treated mice $(\mathbf{a}-\mathbf{d})$. Mice were allowed to freely explore a square shaped arena for $30 \mathrm{~min}$ (imaginarily divided into center and border zone (a)). While there was no difference in the exploratory behavior or in the traveled distance or velocity, there was a significant habituation effect of RD2D3 treated mice to the arena (d). This effect was completely absent in cRD2D3 treated mice (d). Additionally, a Morris water maze (MWM) was performed in which mice were trained for 5 days to find a hidden platform (e). Both treatment groups (RD2D3 and cRD2D3) found the hidden platform faster than placebo treated mice and spent more time in the platform zone during the probe trial (f). Data are shown as mean $\pm \mathrm{SEM}$ (placebo $N=13$, RD2D3 $N=14$ and $c$ RD2D3 $N=12$ ). ${ }^{* *} p<0.01$ and ${ }^{*} p<0.05$. 

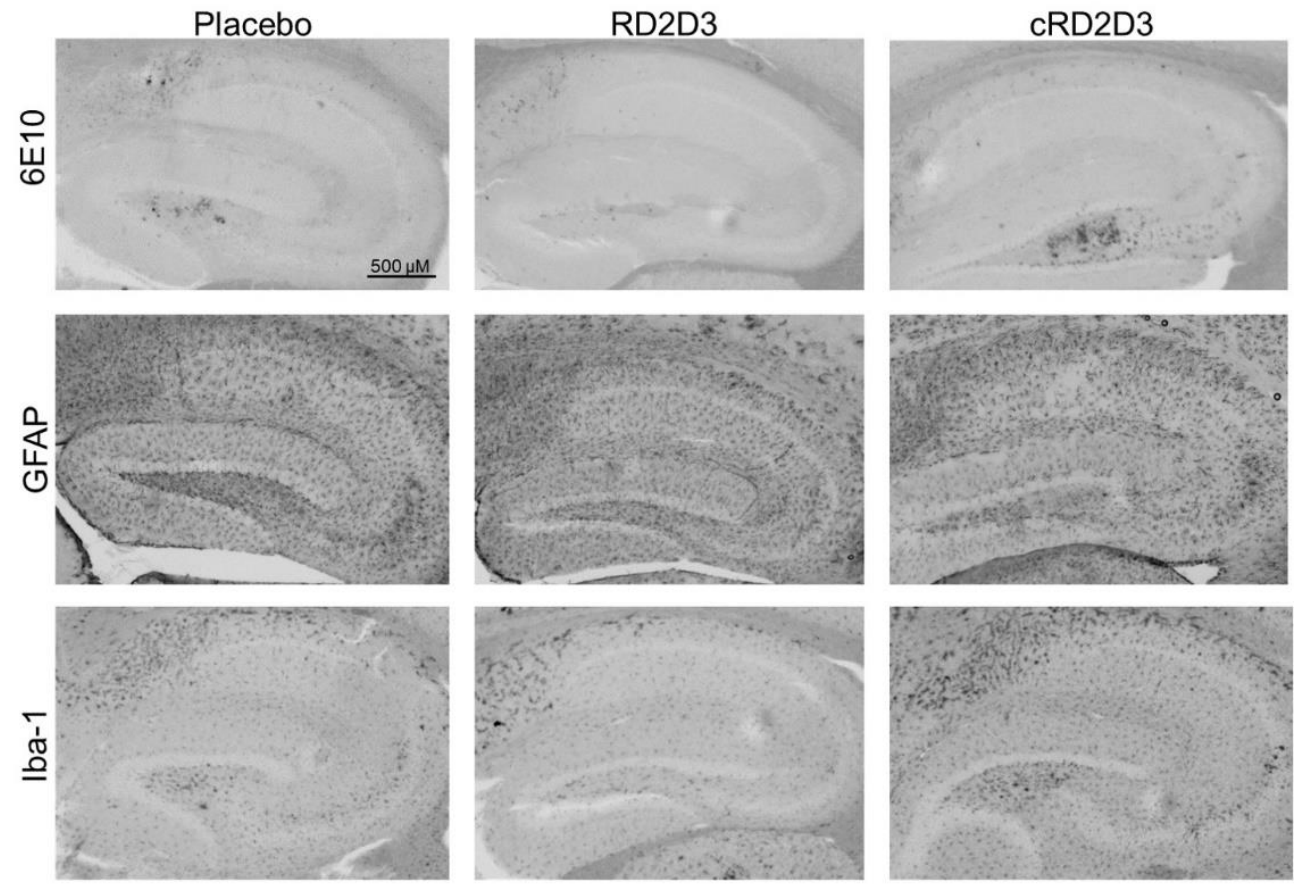

Figure 8. Immunohistochemical analysis of $A \beta$ deposits and neuroinflammation. Representative images of hippocampus of placebo, RD2D3 and cRD2D3 treated mice (placebo $N=13$, RD2D3 $N=14$ and $\mathrm{cRD} 2 \mathrm{D} 3 \mathrm{~N}=12$ ).

\section{Discussion}

More than 100 years have passed since AD was first described by Alois Alzheimer [19]. Meanwhile, many efforts have been made to find a curative or even disease modifying treatment for this devastating neurodegenerative disease [20]. One of the major pathological hallmarks of the disease are deposits of the so called $A \beta$-peptide. No toxic properties are attributed to $A \beta$ in its monomeric, native form. Only a soluble intermediate product, the so-called $A \beta$-oligomers, are considered to be the disease-causing agent $[3,18,21]$. Our group has focused on the development of compounds, which directly destroy toxic $\mathrm{A} \beta$-oligomers. These therapeutic substances are so-called D-peptides, consisting of 12-24 D-enantiomeric amino acid residues. The most promising compound so far is RD2. In the recent years, RD2 has been studied extensively [10-13]. The efficacy of this compound has been demonstrated in several studies in different mouse models, so RD2 is now in the process of proving its efficacy in humans in clinical trials [9]. Out of several drug optimization approaches, the linear tandem-D-peptide RD2D3 and its cyclic equivalent, cRD2D3, came forth [12,14,15,22]. Tandemization and cyclization of the developed D-enantiomeric peptides were postulated to increase the binding affinity to $A \beta(1-42)$, to increase the efficacy to eliminate toxic $A \beta$ oligomers and to increase BBB penetration. This last point in particular was demonstrated by Schartmann et al. [22]. After the pharmacokinetic and some in vitro analyses of the D-peptides were conducted recently, we here performed an in-depth comparison of the in vitro potency of both D-peptides on wild type $A \beta(1-42)$ and on the $D / I A \beta$ mutation and a direct comparison of the D-peptide's in vivo efficacy in Tg-SwDI mice.

During the last years, we could demonstrate that our developed D-peptides bound (mostly) to A $\beta(1-42)$ in the micromolar range. This was also already proven for RD2D3 [14]. However, it was never analyzed if these compounds also bind to different $A \beta$-variants, e.g., the $\mathrm{D} / \mathrm{I}$ mutation, although there have been several treatment studies conducted with a mouse model harboring this and other mutations $[14,16,18,23]$. In this study, we analyzed the binding affinity of both RD2D3 and cRD2D3 to $A \beta(1-42)$ for a direct comparison, and the binding affinity of the abovementioned D-peptides to $D / I A \beta$. The results showed that both D-peptides exhibit similar binding affinities to $A \beta(1-42)$ and $D / I A \beta$. However, this binding is at least twice as strong to $D / I A \beta$ in comparison to $A \beta(1-42)$. 
Taking a closer look into the in vitro functionality of both D-peptides, we performed a ThT and a QIAD assay. Both D-peptides are capable to efficiently inhibit the A $\beta(1-42)$ fibril formation at equimolar concentrations. One might speculate that RD2D3 was slightly more efficient since it also shifted the lag phase of the initial aggregation of $A \beta(1-42)$. By analyzing the ability of both D-peptides to inhibit the $D / I A \beta$ fibril formation, we could not detect a meaningful difference between the potency of both D-peptides. RD2D3 and cRD2D3 inhibit the D/I A $\beta$ fibril formation at equimolar concentrations. Despite the higher binding affinity to $D / I A \beta$ of both D-peptides compared to $A \beta(1-42)$, both D-peptides show a superior potency to inhibit the $A \beta(1-42)$ - than the $D / I A \beta$-fibril formation. $A \beta$-oligomer elimination efficacy was already proven for RD2D3 on A $\beta(1-42)$ [12]. Here, we performed an additional QIAD assay to directly compare the $A \beta$-oligomer elimination efficacy of RD2D3 with cRD2D3. We were able to confirm that RD2D3 and cRD2D3 eliminate A $\beta(1-42)-$ oligomers in vitro especially effective to about $90 \%$ or $80 \%$, respectively. For the first time, we also conducted a QIAD assay with an $\mathrm{A} \beta$ mutation $-\mathrm{D} / \mathrm{I} \mathrm{A} \beta$. We could demonstrate that both D-peptides can eliminate (more) efficiently $\mathrm{D} / \mathrm{I} \mathrm{A} \beta$-oligomers in vitro to about $95 \%$ or $100 \%$, respectively.

In order to verify the already demonstrated proteolytic stability of RD2D3 and cRD2D3 with a more sensitive method (HPLC analysis instead of thin layer chromatography with ${ }^{3} \mathrm{H}$-labeled D-peptide) and to extend the analysis, proteolytic stability tests in different (simulated) human body fluids were done. Analysis of the proteolytic stability of ${ }^{3} \mathrm{H}-$ RD2D3 [15] and ${ }^{3} \mathrm{H}-\mathrm{cRD} 2 \mathrm{D} 3$ [22] in human liver microsomes revealed that both D-peptides appeared to be completely stable. In contrast, the D-peptides were metabolized up to $25 \%$ after $8 \mathrm{~h}$ when analyzed by HPLC. This discrepancy can probably be explained by the fact that analysis by HPLC is many times more sensitive and accurate than analysis by thin-layer chromatography.

In the aforementioned study [22] it was described that cRD2D3 has an extraordinary superior pharmacokinetic profile in C57BL/ 6 mice compared to its linear equivalent RD2D3 after i.v. and i.p. administration $[15,22]$. One of the most outstanding properties of the cyclic D-peptide is its enormously long terminal half-life in plasma of more than 2 days $(58 \mathrm{~h})$. In comparison, the terminal half-life in plasma of RD2D3 was $2.3 \mathrm{~h}$. Moreover, it was shown that cRD2D3 reached its site of action, the brain as a target organ, with concentrations up to four to five times higher than RD2D3.

After analyzing the in vitro profile of the compounds, we wanted to investigate whether the superior pharmacokinetic profile of cRD2D3 was also reflected in improved efficacy in the Tg-SwDI mouse model. For this purpose, we first performed a small inhouse characterization of the mouse model. This served the purpose of confirming that the Tg-SwDI mice also develop the expected (cognitive) deficits in our hands and in our experimental setup. The implemented behavioral experiments (nesting behavior, marble burying, open field test and Morris water maze) gave proof that the Tg-SwDI mouse model develops general and cognitive phenotypic deficits in our hands at 12 months of age. Although development of pathology and especially of cognition impairment is often dependent on breeding cohorts and lab environments, our results are in good accordance with those reported previously [24-27]. After completion of the characterization study, we performed an i.p. treatment study with RD2D3 and cRD2D3 compared to placebo treated mice by use of Alzet osmotic minipumps. Treatment started with 11 months of age and mice were sacrificed after the pump duration of 28 days (at 12 months of age). The treatment start was based on the results of the characterization study, where cognition deficits were detected at 12 months of age. One might speculate that a treatment with an earlier age and a prolonged treatment duration might have also been sufficient. During the last days of treatment, several behavioral tests were conducted. Those tests indicated that RD2D3 had a more pronounced effect on the phenotypic deficits of Tg-SwDI mice than cRD2D3. The analysis of the open field test revealed a clear habituation effect of RD2D3 treated mice to the arena, comparable with the behavior of non-transgenic mice. In contrast, this effect was completely absent in cRD2D3 treated mice. The performed MWM 
suggested that both RD2D3 and cRD2D3 treatment improved the cognitive performance of Tg-SwDI mice compared to placebo treated mice. However, this effect reached significance only in the RD2D3 treated Tg-SwDI mice. The use of a larger number of mice would probably have resulted in this effect also being significant in the cRD2D3 treated TgSwDI mice. Histological analysis of the brains of all mice revealed no difference between the treatment groups, neither on $\mathrm{A} \beta$-deposits nor on activated astrocytes or microglia. One might speculate that a administering a higher dose of the D-peptide concentration or a longer treatment duration would have resulted in a reduction of AD-associated pathology. Previous pharmacokinetic analyses of RD2D3 and cRD2D3 have shown that both D-peptides do reach the brain [6,7]. Summarized, RD2D3 appears to have a superior efficacy to ameliorate the phenotype of Tg-SwDI than cRD2D3 compared to placebo treated mice.

Referring to the hypothesis that cyclized D-peptides also show improved in vivo efficacy compared to linear D-peptides due to a more favorable pharmacokinetic profile [22] could not be confirmed in this study. In all tests performed, cRD2D3 treated Tg-SwDI mice showed similar or slightly worse behavior than RD2D3 treated mice. In conclusion, the superior pharmacokinetic profile of cRD2D3 was not sufficient to translate into improved in vivo efficacies.

\section{Materials and Methods}

\subsection{Peptides}

The D-peptides RD2D3 (ptlhthnrrrrrrprtrlhthrnr) and cRD2D3 (ptlhthnrrrrrrprtrlhthrnr) with amidated C-terminus were purchased as lyophilized powder with $>95 \%$ purity from peptides\&elephants (Henningsdorf, Germany) and Cambridge peptides (UK), respectively.

Synthetic A $\beta(1-42)$ and $\left(\mathrm{Gln}^{22}\right.$, Asn $\left.{ }^{23}\right)$-Amyloid $\beta$-Protein (1-40) $(\mathrm{D} / \mathrm{I} A \beta)$ with $>95 \%$ purity were purchased as lyophilized powder from Bachem (Bubendorf, Switzerland). Lyophilized A $\beta$-species were dissolved overnight in HFIP (1,1,1,3,3,3-hexafluoro-2-propanol, Sigma-Aldrich, Darmstadt, Germany). Aliquots were stored at $-20^{\circ} \mathrm{C}$ until further processing. Before usage, $\mathrm{A} \beta$ was lyophilized and dissolved in $10 \mathrm{mM}$ sodium phosphate buffer, $\mathrm{pH}$ 7.4.

\subsection{In Vitro Potency \\ 4.2.1. Binding Affinity}

The dissociation constant $\left(\mathrm{K}_{\mathrm{D}}\right)$ of RD2D3 and cRD2D3 binding to $\mathrm{A} \beta(1-42)$ and $\mathrm{D} / \mathrm{I}$ $A \beta$ was determined by SPR spectroscopy using a Biacore T200 instrument (Biacore, GE Healthcare, Uppsala, Sweden). A $\beta(1-42)$ or D/I A $\beta$ was used as the ligand, while RD2D3 and cRD2D3 were used as the analyte or vice versa.

By using $A \beta(1-42)$ or $D / I A \beta$ as a ligand, it was immobilized onto a series S CM-5 sensor chip (GE Healthcare, Uppsala, Sweden) by amine coupling. In short, the flow cells were activated by a mixture of $50 \mathrm{mM} \mathrm{N}$-hydroxysuccinimide (NHS) and $16.1 \mathrm{mM} \mathrm{N}$-Ethyl$\mathrm{N}^{\prime}$-(dimethylaminopropyl)carbodiimide (EDC) (XanTec, Düsseldorf, Germany) for $7 \mathrm{~min}$. $\mathrm{A} \beta(1-42)$ or $\mathrm{D} / \mathrm{I} \mathrm{A} \beta$ were dissolved to a final concentration of $50 \mu \mathrm{g} / \mathrm{mL}$ in $10 \mathrm{mM}$ sodium acetate $\mathrm{pH} 5$ (AppliChem, Darmstadt, Germany) and injected over one of the activated flow cells to a final signal of $1700 \mathrm{RU}$. After the immobilization, the ligand and reference flow cells were quenched by injecting $1 \mathrm{M}$ ethanolamine $\mathrm{pH} 8.5$ (XanTec, Düsseldorf, Germany) for $7 \mathrm{~min}$. For the determination of the $\mathrm{K}_{\mathrm{D}}$, multicycle kinetic experiments were performed with $10 \mathrm{mM}$ HEPES + $50 \mathrm{mM} \mathrm{NaCl}$ (AppliChem, Darmstadt, Germany) pH 7.4 as the running buffer at $25^{\circ} \mathrm{C}$ and at a flow rate of $30 \mu \mathrm{L} / \mathrm{min}$. The peptides were diluted in the running buffer to the following concentrations: $20 \mu \mathrm{M}, 10 \mu \mathrm{M}, 5 \mu \mathrm{M}, 2.5 \mu \mathrm{M}, 1.25 \mu \mathrm{M}$ and $0.625 \mu \mathrm{M}$. All samples were injected over the flow cells for $180 \mathrm{~s}$, followed by a dissociation step of $600 \mathrm{~s}$ with running buffer. Regeneration of the sensor chip was accomplished by a $45 \mathrm{~s}$ injection of $2 \mathrm{M}$ of guanidinium hydrochloride (AppliChem, Darmstadt, Germany). The reference flow cell and buffer injections $(c=0 \mathrm{nM})$ were used for double referencing 
of the sensorgrams. For data evaluation, the sensorgrams were fitted by the steady-state affinity model implemented in the Biacore T200 Evaluation Software 3.2.

By using the compounds (RD2D3 and cRD2D3) as ligands, they were immobilized on two separate channels on a series S CM-5 sensor chip (Cytiva, GE Healthcare, Uppsala, Sweden) by amine coupling. Both flow cells on each channel were activated with a mixture of $50 \mathrm{mM}$ N-hydroxysuccinimide (NHS) and $16.1 \mathrm{mM} \mathrm{N}$-ethyl-N'-(dimethylaminopropyl)carbodiimide (EDC) (XanTec, Düsseldorf, Germany) for $7 \mathrm{~min}$. The peptides were diluted to $50 \mu \mathrm{g} / \mathrm{mL}$ in $10 \mathrm{mM}$ maleic acid pH 6 (AppliChem, Darmstadt, Germany) and injected over flow cell two of each channel to a final signal between 600 and 900 RU. After the peptides were immobilized, the ligand and reference flow cells of each channel were quenched by injecting $1 \mathrm{M}$ ethanolamine $\mathrm{pH} 8.5$ (XanTec, Düsseldorf, Germany) for $7 \mathrm{~min}$. For the determination of the $K_{D}$ multicycle kinetic experiments were performed with $10 \mathrm{mM}$ HEPES + $50 \mathrm{mM} \mathrm{NaCl}$ (AppliChem, Darmstadt, Germany) pH 7.4 as the running buffer at $25^{\circ} \mathrm{C}$ and at a flow rate of $30 \mu \mathrm{L} / \mathrm{min}$. A $\beta(1-42)$ or $\mathrm{D} / \mathrm{I} A \beta$ were diluted in the running buffer to the following concentrations: $10 \mu \mathrm{M}, 3.3 \mu \mathrm{M}, 1.1 \mu \mathrm{M}, 0.37 \mu \mathrm{M}, 0.12 \mu \mathrm{M}, 0.045 \mu \mathrm{M}$, $0.014 \mu \mathrm{M}$ and $0.0045 \mu \mathrm{M}$. All samples were injected over the flow cells for $180 \mathrm{~s}$, followed by a dissociation step of $600 \mathrm{~s}$ with running buffer. Regeneration of the sensor chip was accomplished by a $45 \mathrm{~s}$ injection of $2 \mathrm{M}$ guanidinium hydrochloride (AppliChem, Darmstadt, Germany). The reference flow cell of each channel and the buffer injections $(\mathrm{c}=0 \mathrm{nM})$ were used for double referencing of the sensorgrams. For data evaluation, the sensorgrams were fitted by the steady-state affinity model implemented in the Biacore Insight Evaluation Software version 3.0.

\subsubsection{Thioflavin-T Assay}

Using the ThT assay, the potency of RD2D3 and cRD2D3 to inhibit the fibril formation of $A \beta(1-42)$ and $D / I A \beta$ was analyzed. For this purpose, $10 \mu \mathrm{M} A \beta(1-42)$ or $D / I A \beta$ were incubated with $10 \mu \mathrm{M}$ of the corresponding D-peptide and $5 \mu \mathrm{M}$ ThT. ThT fluorescence was monitored over $24 \mathrm{~h}$ every $6 \mathrm{~min}$ at $\lambda \mathrm{ex}=440 \mathrm{~nm}$ and $\lambda \mathrm{em}=490 \mathrm{~nm}$ in a fluorescence plate reader (Clariostar, BMG Labtech, Ortenberg, Germany) at RT. Correction was done using all supplements without $\mathrm{A} \beta$ and D-peptide (blank).

\subsubsection{QIAD Assay}

According to Brener et al., a QIAD assay was performed in order to evaluate the $\mathrm{A} \beta(1-$ 42) or $\mathrm{D} / \mathrm{I} A \beta$ oligomer elimination efficacies of RD2D3 and cRD2D3 [18]. In short, $80 \mu \mathrm{M}$ lyophilized $A \beta(1-42)$ or $40 \mu \mathrm{M} \mathrm{D} / \mathrm{I} A \beta$ was preincubated for $2 \mathrm{~h}$ or 15 min, respectively, to enrich $A \beta$-oligomers. Afterwards, either $20 \mu \mathrm{M}$ RD2D3 or cRD2D3 for A $\beta(1-42)$ or $10 \mu \mathrm{M}$ RD2D3 or cRD2D3 for $\mathrm{D} / \mathrm{I} \mathrm{A} \beta$ were added to the preincubated solution and coincubated for additional $30 \mathrm{~min}$. Subsequently, the samples were loaded on the top of a density gradient (5-50\% $(w / v)$ iodixanol (OptiPrep, Sigma-Aldrich, Darmstadt, Germany)) followed by an ultracentrifugation step $\left(3 \mathrm{~h}\right.$ at $4{ }^{\circ} \mathrm{C}$ and $259000 \times g$ (Optima TL-100, Beckman Coulter, Brea, CA, USA)). Following the ultracentrifugation, 14 fractions (140 $\mu \mathrm{L}$ each) were harvested by upward displacement. Fraction 15 contains the dissolved pellet in $6 \mathrm{M}$ guanidine hydrochloride solution. Top fractions 1-2 contained $\mathrm{A} \beta$-monomers, fractions 4-6 contained the $A \beta$-oligomers, which are of special interest, and the bottom fractions 11-14 contained high molecular weight (co-)precipitates or aggregated $A \beta$. A $\beta(1-$ 42) or D/I A $\beta$ concentrations of each fraction were determined via analytical RP-HPLC and UV absorbance detection at $214 \mathrm{~nm}$.

\subsubsection{Proteolytic Stability}

Both compounds have already been extensively studied with respect to their in vivo pharmacokinetic profiles $[15,22]$. Here, we performed some additional test to investigate the proteolytic stability of the compounds in simulated gastric (SGF) and intestinal fluid (SIF) and human plasma and human liver microsomes. The experiments were performed as described previously [28]. According to the guidelines of the European Pharmacopoeia 
7.0, SGF and SIF were prepared. Plasma was purchased from Biotrend with K3-EDTA as an anticoagulant (Biotrend, Köln, Germany). Human liver microsomes were pooled from different donors and purchased from Sekisui XenoTech (Kansas City, KS, USA).

In order to determine whether the compounds RD2D3 and cRD2D3 are stable or would be metabolized, $150 \mu \mathrm{M}$ of each compound was incubated in triplicate for defined time points slightly shaking at $37^{\circ} \mathrm{C}$ in the media described above (SGF and SIF: 4 and $8 \mathrm{~h}$, plasma: 8, 24 and $48 \mathrm{~h}$ and microsomes: 8 and $24 \mathrm{~h}$ ). Incubation was stopped by precipitating the proteins. For this, $3 \%$ trichloroacetic acid $(w / v)$ was added to the respective sample under vortexing followed by a centrifugation step $\left(14,000 \times g\right.$ at $4{ }^{\circ} \mathrm{C}$ for $5 \mathrm{~min}$ ). For analysis, supernatants containing the compounds were collected. The precipitated media without compound was used as a control and as reference for the quantification served each medium with compound where the reaction was stopped immediately by precipitating the proteins. All samples were analyzed by RP-HPLC as previously described [28].

\subsection{In Vivo Efficacy}

\subsubsection{Animals}

In this study, the Tg-SwDI AD mouse model was used. Tg-SwDI were first described by Davis et al. in 2004 and carried the human APP gene (isoform 770) with the Swedish (K670N/M671L), Dutch (E693Q) and Iowa (D694N) mutations under the control of the mouse Thy1 promoter [24]. A $\beta$-depositions can be found starting with three months of age and cognition deficits can be detected as early as three months of age in the Barnes maze $[24,26]$. Tg-SwDI mice are known to show distinct AD-associated pathology. This includes $A \beta$-deposition in various areas of the brain (particularly in the cortex and hippocampus) and associated gliosis. Gliosis is characterized by the presence of activated microglia and astrocytes, especially in those regions where $A \beta$-deposits are also found. In addition to $A \beta$-deposits in the tissue, this mouse model is particularly characterized by a so-called cerebral amyloid angiopathy (CAA). This means that Tg-SwDI mice show increased $A \beta$-deposits in cerebral vessels.

Tg-SwDI mice were ordered by the Jackson Laboratory (C57BL/6-Tg(Thy1-APPSwDutIowa)BWevn/Mmjax, Jackson Laboratory, Bar Harbor, ME, USA) and bred in-house in a controlled environment on a light/dark cycle (12/12 h), with $54 \%$ humidity and a temperature of $22^{\circ} \mathrm{C}$. Food and water were available ad libitum.

\subsubsection{Ethical Approval}

All animal experiments were done in accordance with the German Law on the protection of animals and approved by the Landesamt für Natur, Umwelt und Verbraucherschutz (LANUV) North-Rhine-Westphalia, Germany (AZ84-02.04.2016.A523).

\subsubsection{Characterization}

In order to perform a short in-house characterization of the Tg-SwDI mouse model, we used 12 months old female homozygous $(n=12)$ and corresponding wild type $(n=13)$ mice to verify cognition deficits at the same age at which the treatment study should be performed. Mice were tested in several behavioral tests to identify differences between Tg-SwDI and WT mice.

\subsubsection{Nesting Behavior}

Building a nest is a core behavior of mice, not just for maternal nesting. It is crucial for mice for shelter from the environment and protection against predators. Here, a protocol after Deacon was used. In brief, $1 \mathrm{~h}$ before the dark phase of the animal house, mice were single placed in a new cage with a fresh nestlet (Sniff, Soest, Germany). The next morning, built nests were scored from 1 to 5 , whereby 1 represents no nest and 5 represents a perfect nest [29]. 


\subsubsection{Marble Burying}

Digging and burrowing is a fundamental behavior of mice to find and hide food and to build a nest. Several drugs or genetic modifications may alter the digging behavior of mice. For analysis of digging behavior, the marble burying test was performed after Deacon. Mice were single placed in a new cage with approximately $5 \mathrm{~cm}$ deep wood chip bedding. On the bedding, 12 glass marbles (diameter: $1.6 \mathrm{~cm}$ and weight: $5.3 \mathrm{~g}$ ) were laid down in a predefined pattern. After $30 \mathrm{~min}$, the number of marbles buried was counted [30].

\subsubsection{Open Field Test}

Analysis of explorative and anxiety related behavior of mice can be done by use of the so-called open field test [31]. Following a $30 \mathrm{~min}$ habituation phase in the experiment's room, the mice were placed in a square-shaped arena $(45 \mathrm{~cm} \times 45 \mathrm{~cm} \times 45 \mathrm{~cm})$ for an additional $30 \mathrm{~min}$, imaginarily divided into two zones: center and border zone (center: $19 \mathrm{~cm} \times 19 \mathrm{~cm}$ and border: space around the center zone). During the $30 \mathrm{~min}$ exploration, mice were recorded with a camera driven tracking system (Ethovision 15, Noldus, Wageningen, The Netherlands). For analysis, the duration the mice stayed in each zone was evaluated. Moreover, several time slots (1: 0-5 min, 2: 5-10 min, 3: 10-15 min, 4: 15-20 min and 5: $20-25 \mathrm{~min}$ ) were analyzed independently to determine habituation behavior of the mice.

\subsubsection{Morris Water Maze}

Investigation of spatial learning can be conducted by the use of the MWM. The MWM is one of the most widely used tests to investigate cognitive impairments, especially spatial memory, in neuroscience [32]. The water maze we have used consists of a circular white pool $(120 \mathrm{~cm}$ in diameter and $60 \mathrm{~cm}$ in height). Rendering of the water was ensured by adding a non-toxic white coloring solution. To conduct the test, the pool is imaginarily subdivided into four quadrants (north-east, south-east, south-west and north-west). In the middle of the target quadrant, an invisible round platform was placed $1 \mathrm{~cm}$ below the surface. The used protocol was modified after the original one from Morris et al. [33]. During the training period, mice were allowed to swim for $60 \mathrm{~s}$ or until they found the hidden platform. If they did not find the hidden platform, they were set on the platform for $10 \mathrm{~s}$ to orient themselves before they were returned to their cages. On each of the five training days, the mice had to swim four trials, each trial starting from a different quadrant, with the order changing each day. To avoid a decrease of body temperature of the mice, they were placed under a heating lamp for $60 \mathrm{~s}$ between each trial. On the sixth day, the mice had to swim freely without a hidden platform (probe trial). During each trial, the mice were recorded with a video driven tracking system (Ethovision 15, Noldus, Wageningen, The Netherlands). The following parameters were analyzed: escape latency to find the hidden platform during the training phase or duration in platform zone during the probe trial.

\subsection{Treatment}

Eleven months old Tg-SwDI were treated for 28 d i.p. by use of an osmotic minipump (Alzet osmotic pumps, Modell 1004, Charles River, Wilmington, MS, USA) with a daily dosage of $8 \mathrm{mg} / \mathrm{kg}$ peptide (RD2D3 $(n=14)$ or cRD2D3 $(n=12))$ in PBS (pH 7.4) or vehicle (Placebo $(n=13)$, PBS, $\mathrm{pH} 7.4)$. The minipumps and the i.p. application route have been used to achieve continuous application of the study drug. After surgery, mice were monitored daily the following three days and twice each week until the end of the treatment. A loss of body weight and severe conspicuities were defined as exclusion criteria. No mouse was affected by these exclusion criteria. During the last one and a half weeks of treatment, different behavioral tests (nesting behavior, marble burying, open field test and MWM) were conducted with all mice. 
Since RD2D3 did not show any efficacy in vivo after oral administration [12], but after i.p. administration [14], we decided to perform an i.p. treatment study for the direct comparison of the linear and cyclic D-peptide.

\subsection{Histology}

After the treatment duration was finished, the mice were anesthetized (intraperitoneal injection of $100 \mathrm{mg} / \mathrm{kg}$ ketamine (bela-pharm, Vechta, Germany) and $0.3 \mathrm{mg} / \mathrm{kg}$ medetomidine (Dormilan, alfavet, Neumünster, Germany) and transcardially perfused with ice-cold PBS. Subsequently, brains were removed and one hemisphere was frozen. The other hemisphere was fixed overnight in $4 \%$ paraformaldehyde, followed by a post-fixation and cryoprotection in 30\% sucrose for an additional day. For histological analysis, $40 \mu \mathrm{m}$ free floating sections were prepared on a cryostat. In total, eight series of six sections each were cut sagittally through the brain. One series of each brain was used for the following stainings: 6E10 (Biolegend, San Diego, CA, USA) for A $\beta$-deposits, GFAP (Agilent, Santa Clara, CA, USA) for reactive astrocytes and Iba-1 (Fujifilm, Neuss, Germany) for activated microglia. In brief, sections were washed in TBST (TBS with 1\% Triton X-100), followed by an overnight incubation with the primary antibody $(6 \mathrm{E} 101 \mu \mathrm{g} / \mathrm{mL}$, GFAP $1 \mu \mathrm{g} / \mathrm{mL}$ and Iba- $10.5 \mu \mathrm{g} / \mathrm{mL}$ ). The very next day, sections were washed in TBST, followed by the incubation with the secondary antibody (6E10 goat anti-Mouse $\operatorname{IgG}(\mathrm{H}+\mathrm{L})$ secondary antibody $1.3 \mu \mathrm{g} / \mathrm{mL}$, GFAP and Iba-1 goat anti-rabbit IgG $(\mathrm{H}+\mathrm{L})$ secondary antibody, $1.5 \mu \mathrm{g} / \mathrm{mL}$, all Thermo Fisher, Germany) for $2 \mathrm{~h}$ at RT. After an additional washing step in TBST, stainings were visualized with the use of 3,3' diaminobenzidine (DAB) enhanced with saturated nickel ammonium sulphate solution. Afterwards, sections were mounted with DPX Mountant (Sigma-Aldrich, Darmstadt, Germany).

To avoid any irregularities in the staining results, all stainings were performed in one batch and in one microscopy session. Zeiss SteREO Lumar V12 microscope and the according software (Zeiss AxioVision 6.4 RE) was used for visualization. Quantification was done with Image (NIH, USA) and CellProfiler (Broad Institute, Cambridge, MA, USA).

\subsection{Statistics}

All statistical analyses were performed using GraphPad Prism 8 (GraphPad Software, Inc., San Diego, CA, USA) or SigmaPlot Version 11 (Systat Software, Düsseldorf, Germany). In vitro data are represented as mean $\pm \mathrm{SD}$ and in vivo data as mean $\pm \mathrm{SEM}$. Normal distributed data were analyzed by use of an unpaired one- or two-tailed $t$-test or one-way analysis of variance (ANOVA) with Tukey post hoc analysis. Not normal distributed data were analyzed by use of the Kruskal-Wallis test with Dunn's multiple comparison test. The MWM and open field test were analyzed by a repeated measure ANOVA with Fisher or Bonferroni post hoc analysis. Data with $p<0.05$ were stated as significant.

\section{Conclusions}

In this study, we analyzed the in vitro potency of two of our developed D-enantiomeric D-peptides on an artificial contribution of two familial mutations within the $A \beta$ sequence, namely the Dutch and Iowa mutations, for the first time. The analysis of the in vitro profile revealed that RD2D3 and cRD2D3 have a similar potency on both analyzed A $\beta$-species, regarding the potency to inhibit the $A \beta$-fibril formation and the potency to eliminate toxic $\mathrm{A} \beta$-oligomers. Referring to the hypothesis that a cyclization of our D-peptides might show superior in vivo efficacy could not be confirmed in this study. Compared to linear RD2D3, cyclic cRD2D3 failed to show superior efficacy.

Author Contributions: The overall study was planned by S.S. and D.W. In vivo study was planned by S.S., D.W., J.K., and A.W. In vitro experiments were carried out by I.G. and S.S. In vivo experiments were done by D.H. and L.C.C. Histological analysis were done by D.H. and S.S. Data were analyzed by S.S. All authors contributed to writing. All authors have read and agreed to the published version of the manuscript. 
Funding: D.W. was supported by grants from the Russian Science Foundation (RSF) (project no. 20-64-46027), by the Technology Transfer Fund of the Forschungszentrum Jülich and was supported by "Portfolio Drug Research" of the "Impuls und Vernetzungs-Fonds der Helmholtzgemeinschaft.

Institutional Review Board Statement: All animal experiments were performed in accordance with the German Law on the protection of animals (TierSchG $\S \S 7-9$ ) and were approved by a local ethics committee (LANUV, North-Rhine-Westphalia, Germany, reference number: Az: 84-02.04.2016.A523).

Data Availability Statement: The data presented in this study are available in this article.

Conflicts of Interest: D.W. declares to be the coinventor of patent applications describing the compounds used in the study. D.W. declares to be the cofounder and co-owner of the company Priavoid $\mathrm{GmbH}$. This had no influence on the interpretation of the data. All other authors declare no conflict of interest.

\section{References}

1. Grande, G.; Qiu, C.; Fratiglioni, L. Prevention of dementia in an ageing world: Evidence and biological rationale. Ageing Res. Rev. 2020, 64, 101045. [CrossRef]

2. Hardy, J.; Selkoe, D.J. The amyloid hypothesis of Alzheimer's disease: Progress and problems on the road to therapeutics. Science 2002, 297, 353-356. [CrossRef] [PubMed]

3. Selkoe, D.J.; Hardy, J. The amyloid hypothesis of Alzheimer's disease at 25 years. EMBO Mol. Med. 2016, 8, 595-608. [CrossRef] [PubMed]

4. Cline, E.N.; Bicca, M.A.; Viola, K.L.; Klein, W.L. The Amyloid- $\beta$ Oligomer Hypothesis: Beginning of the Third Decade. J. Alzheimer's Dis. 2018, 64, S567-S610. [CrossRef] [PubMed]

5. Willbold, D.; Kutzsche, J. Do We Need Anti-Prion Compounds to Treat Alzheimer's Disease? Molecules 2019, 24, 2237. [CrossRef]

6. van Groen, T.; Wiesehan, K.; Funke, S.A.; Kadish, I.; Nagel-Steger, L.; Willbold, D. Reduction of Alzheimer's disease amyloid plaque load in transgenic mice by D3, A D-enantiomeric peptide identified by mirror image phage display. ChemMedChem 2008, 3, 1848-1852. [CrossRef] [PubMed]

7. Van Regenmortel, M.H.; Muller, S. D-peptides as immunogens and diagnostic reagents. Curr. Opin. Biotechnol. 1998, 9, 377-382. [CrossRef]

8. Dintzis, H.M.; Symer, D.E.; Dintzis, R.Z.; Zawadzke, L.E.; Berg, J.M. A comparison of the immunogenicity of a pair of enantiomeric proteins. Proteins Struct. Funct. Bioinform. 1993, 16, 306-308. [CrossRef]

9. Kutzsche, J.; Jürgens, D.; Willuweit, A.; Adermann, K.; Fuchs, C.; Simons, S.; Windisch, M.; Hümpel, M.; Rossberg, W.; Wolzt, M.; et al. Safety and pharmacokinetics of the orally available antiprionic compound PRI-002: A single and multiple ascending dose phase I study. Alzheimer Dement. Transl. Res. Clin. Interv. 2020, 6, e12001. [CrossRef]

10. van Groen, T.; Schemmert, S.; Brener, O.; Gremer, L.; Ziehm, T.; Tusche, M.; Nagel-Steger, L.; Kadish, I.; Schartmann, E.; Elfgen, A.; et al. The Abeta oligomer eliminating D-enantiomeric peptide RD2 improves cognition without changing plaque pathology. Sci. Rep. 2017, 7, 16275. [CrossRef]

11. Schemmert, S.; Schartmann, E.; Zafiu, C.; Kass, B.; Hartwig, S.; Lehr, S.; Bannach, O.; Langen, K.; Shah, N.J.; Kutzsche, J.; et al. Abeta Oligomer Elimination Restores Cognition in Transgenic Alzheimer's Mice with Full-blown Pathology. Mol. Neurobiol. 2019, 56, 2211-2223. [CrossRef]

12. Kutzsche, J.; Schemmert, S.; Tusche, M.; Neddens, J.; Rabl, R.; Jürgens, D.; Brener, O.; Willuweit, A.; Hutter-Paier, B.; Willbold, D. Large-Scale Oral Treatment Study with the Four Most Promising D3-Derivatives for the Treatment of Alzheimer's Disease. Molecules 2017, 22, 1693. [CrossRef]

13. Schemmert, S.; Schartmann, E.; Honold, D.; Zafiu, C.; Ziehm, T.; Langen, K.-J.; Shah, N.J.; Kutzsche, J.; Willuweit, A.; Willbold, D. Deceleration of the neurodegenerative phenotype in pyroglutamate-Abeta accumulating transgenic mice by oral treatment with the Abeta oligomer eliminating compound RD2. Neurobiol. Dis. 2019, 124, 36-45. [CrossRef] [PubMed]

14. Cavini, I.A.; Munte, C.E.; Erlach, M.B.; van Groen, T.; Kadish, I.; Zhang, T.; Ziehm, T.; Nagel-Steger, L.; Kutzsche, J.; Kremer, W.; et al. Inhibition of amyloid $A \beta$ aggregation by high pressures or specific d-enantiomeric peptides. Chem. Commun. 2018, 54, 3294-3297. [CrossRef] [PubMed]

15. Leithold, L.H.E.; Jiang, N.; Post, J.; Niemietz, N.; Schartmann, E.; Ziehm, T.; Kutzsche, J.; Shah, N.J.; Breitkreutz, J.; Langen, K.-J.; et al. Pharmacokinetic properties of tandem d-peptides designed for treatment of Alzheimer's disease. Eur. J. Pharm. Sci. 2016, 89, 31-38. [CrossRef] [PubMed]

16. Ziehm, T.; Brener, O.; Van Groen, T.; Kadish, I.; Frenzel, D.; Tusche, M.; Kutzsche, J.; Reiß, K.; Gremer, L.; Nagel-Steger, L.; et al. Increase of Positive Net Charge and Conformational Rigidity Enhances the Efficacy of d-Enantiomeric Peptides Designed to Eliminate Cytotoxic A $\beta$ Species. ACS Chem. Neurosci. 2016, 7, 1088-1096. [CrossRef]

17. Schartmann, E.; Schemmert, S.; Niemietz, N.; Honold, D.; Ziehm, T.; Tusche, M.; Elfgen, A.; Gering, I.; Brener, O.; Shah, N.J.; et al. In Vitro Potency and Preclinical Pharmacokinetic Comparison of All-D-Enantiomeric Peptides Developed for the Treatment of Alzheimer's Disease. J. Alzheimer Dis. 2018, 64, 859-873. [CrossRef] 
18. Brener, O.; Dunkelmann, T.; Gremer, L.; Van Groen, T.; Mirecka, E.A.; Kadish, I.; Willuweit, A.; Kutzsche, J.; Jürgens, D.; Rudolph, S.; et al. QIAD assay for quantitating a compound's efficacy in elimination of toxic A $\beta$ oligomers. Sci. Rep. 2015, 5, 13222. [CrossRef]

19. Alzheimer, A. Concerning unsual medical cases in old age. Z. Gesamte Neurol. Psychiatr. 1911, 4, 356-385. [CrossRef]

20. Scheltens, P.P.; De Strooper, B.; Kivipelto, M.; Holstege, H.; Chételat, G.; Teunissen, C.E.; Cummings, J. Alzheimer's disease. Lancet 2021, 397, 1577-1590. [CrossRef]

21. Li, S.; Selkoe, D.J. A mechanistic hypothesis for the impairment of synaptic plasticity by soluble A $\beta$ oligomers from Alzheimer's brain. J. Neurochem. 2020, 154, 583-597. [CrossRef]

22. Schartmann, E.; Schemmert, S.; Ziehm, T.; Leithold, L.H.E.; Jiang, N.; Tusche, M.; Shah, N.J.; Langen, K.-J.; Kutzsche, J.; Willbold, D.; et al. Comparison of blood-brain barrier penetration efficiencies between linear and cyclic all-d-enantiomeric peptides developed for the treatment of Alzheimer's disease. Eur. J. Pharm. Sci. 2018, 114, 93-102. [CrossRef]

23. Klein, A.N.; Ziehm, T.; van Groen, T.; Kadish, I.; Elfgen, A.; Tusche, M.; Thomaier, M.; Reiss, K.; Brener, O.; Gremer, L.; et al. Optimization of d-Peptides for Abeta Monomer Binding Specificity Enhances Their Potential to Eliminate Toxic Abeta Oligomers. ACS Chem. Neurosci. 2017, 8, 1889-1900. [CrossRef]

24. Davis, J.; Xu, F.; Deane, R.; Romanov, G.; Previti, M.L.; Zeigler, K.; Zlokovic, B.V.; van Nostrand, W.E. Early-onset and robust cerebral microvascular accumulation of amyloid beta-protein in transgenic mice expressing low levels of a vasculotropic Dutch/Iowa mutant form of amyloid beta-protein precursor. J. Biol. Chem. 2004, 279, 20296-20306. [CrossRef] [PubMed]

25. Miao, J.; Xu, F.; Davis, J.; Otte-Höller, I.; Verbeek, M.M.; van Nostrand, W.E. Cerebral microvascular amyloid beta protein deposition induces vascular degeneration and neuroinflammation in transgenic mice expressing human vasculotropic mutant amyloid beta precursor protein. Am. J. Pathol. 2005, 167, 505-515. [CrossRef]

26. Xu, F.; Grande, A.M.; Robinson, J.K.; Previti, M.L.; Vasek, M.; Davis, J.; van Nostrand, W.E. Early-onset subicular microvascular amyloid and neuroinflammation correlate with behavioral deficits in vasculotropic mutant amyloid beta-protein precursor transgenic mice. Neuroscience 2007, 146, 98-107. [CrossRef] [PubMed]

27. Robison, L.S.; Francis, N.; Popescu, D.L.; Anderson, M.E.; Hatfield, J.; Xu, F.; Anderson, B.J.; Van Nostrand, W.E.; Robinson, J.K. Environmental Enrichment: Disentangling the Influence of Novelty, Social, and Physical Activity on Cerebral Amyloid Angiopathy in a Transgenic Mouse Model. Int. J. Mol. Sci. 2020, 21, 843. [CrossRef] [PubMed]

28. Elfgen, A.; Santiago-Schübel, B.; Gremer, L.; Kutzsche, J.; Willbold, D. Surprisingly high stability of the A $\beta$ oligomer eliminating all-d-enantiomeric peptide D3 in media simulating the route of orally administered drugs. Eur. J. Pharm. Sci. 2017, 107, 203-207. [CrossRef]

29. Deacon, R.M.J. Assessing nest building in mice. Nat. Protoc. 2006, 1, 1117-1119. [CrossRef] [PubMed]

30. Deacon, R.M.J. Digging and marble burying in mice: Simple methods for in vivo identification of biological impacts. Nat. Protoc. 2006, 1, 122-124. [CrossRef]

31. Archer, J. Tests for emotionality in rats and mice: A review. Anim. Behav. 1973, 21, 205-235. [CrossRef]

32. Paulson, J.B.; Ramsden, M.; Forster, C.; Sherman, M.A.; McGowan, E.; Ashe, K.H. Amyloid plaque and neurofibrillary tangle pathology in a regulatable mouse model of Alzheimer's disease. Am. J. Pathol. 2008, 173, 762-772. [CrossRef] [PubMed]

33. Morris, R.G.M.; Garrud, P.; Rawlins, J.N.P.; O'Keefe, J. Place navigation impaired in rats with hippocampal lesions. Nature 1982, 297, 681-683. [CrossRef] [PubMed] 